\section{Possible role for leptin in treating depression}

Most studies investigating the adipocyte hormone, leptin, have focused on its appetiteregulating activity in the hypothalamus. Leptin receptors are also found in brain structures involved in emotional and mood regulation, however, such as the hippocampus. Support for a possible role of leptin in modulating mood is provided by a new animal study, which found that plasma leptin levels of rats chronically subjected to social defeat or unpredictable stress were lower than those of controls. Leptin injections reversed the 'anhedonic' behaviors of these rats.

The antidepressant-like effects of leptin were evaluated by monitoring sucrose preference and by the forced-swimming test. In both tests, leptin's effects were comparable to those of conventional antidepressants. Interestingly, however, different behaviors were induced by leptin and the antidepressant desipramine, respectively, in the forced-swimming test: desipramine was associated with climbing activity whereas leptin was associated with longer duration of swimming. This difference was not attributable to leptin's energy-regulating activity; comparison of the effects of infusing leptin directly into different brain areas revealed that hippocampal infusion produced similar antidepressant-like effects to systemic treatment, whereas hypothalamic infusion produced no such effects.

Rising plasma leptin levels have been observed in patients who were responding to conventional antidepressant treatment, although depressed patients do not always have lower leptin levels than healthy individuals. It might be that leptin levels are low only in a subset of depressed patients.

Caroline Barranco

Original article Lu X-Y et al. (2006) Leptin: a potential novel antidepressant. Proc Natl Acad Sci USA 103: 1593-1598

\section{Vitamin D supplementation given to pregnant women could enhance child bone mass}

In this longitudinal study, Javaid et al. tested the hypothesis that vitamin D insufficiency in mothers during pregnancy causes effects on their offspring's bone mass-a relationship that has not previously been directly assessed.
Overall, 198 children born in a Southampton, UK hospital during 1991 and 1992 took part in this study. During late pregnancy, concentrations of the mothers' $25(\mathrm{OH})$-vitamin D were measured, and their weight, height and information on dietary supplementation were also recorded. After delivery, the babies were weighed and a sample of the umbilical venous blood was analyzed. The children's bone mass was measured at 9-year follow-up.

Mothers who were deficient in vitamin D during late pregnancy had children with a reduced whole-body bone mineral content, bone area and areal BMD at 9 years of age. Height and weight of children at 9 years was not associated with levels of maternal vitamin $D$ during late pregnancy. Predictors of maternal vitamin $D$ concentrations were estimated exposure to ultraviolet-B light and use of vitamin D supplementation. Umbilical concentrations of calcium correlated positively with whole-body bone mineral content of the child at 9 years.

The mechanisms by which maternal vitamin $D$ insufficiency affects the child's bone mass remain unknown; however, the authors suggest that these might involve an impairment of placental calcium transport. The authors conclude that vitamin $\mathrm{D}$ supplementation given to pregnant women (particularly if their last trimester is during the winter months) could enhance fetal bone mineral accrual and reduce osteoporotic fracture in their offspring later in life.

Marie Lofthouse

Original article Javaid MK et al. (2006) Maternal vitamin $\mathrm{D}$ status during pregnancy and childhood bone mass at age 9 years: a longitudinal study. Lancet 367: 36-43

\section{Efficacy of raloxifene and teriparatide in patients with osteoporosis}

Earlier studies showed that raloxifene-a selective estrogen-receptor modulator-reduced the risk of vertebral fracture in postmenopausal women; however, a systematic analysis that included the results of some of the newer trials showing the efficacy of raloxifene had not been carried out. Seeman et al., therefore, performed a meta-analysis that included all randomized, double-blind, placebo-controlled trials of raloxifene of at least 1 year in duration, which recorded vertebral fracture data obtained from spinal radiographs in women. In total, seven 